\title{
Synergistic favorable effect of eicosapentaenoic acid and statin on atherosclerosis.
}

\author{
Takahito Yuki ${ }^{1}$, Masaaki Ii $^{2 *}$, Takehiro Nagatsuka², Tomohiro Fujisaka ${ }^{3}$, Yoshihiro Takeda ${ }^{3}$, Masaaki \\ Hoshiga $^{3}$, Akihisa Imagawa ${ }^{1}$ \\ ${ }^{1}$ First Department of Internal Medicine, Faculty of Medicine, Osaka Medical College, Osaka, Japan \\ ${ }^{2}$ Division of Research Animal Laboratory and Translational Medicine, Research and Development Center, Osaka \\ Medical College, Osaka, Japan \\ ${ }^{3}$ Third Department of Internal Medicine, Faculty of Medicine, Osaka Medical College, Osaka, Japan
}

\begin{abstract}
Background and aims: Combination of Eicosapentaenoic Acid (EPA) and HMG-CoA reductase inhibitor (statin) are known to be an effective therapy for cardiovascular diseases, however, their biological roles in the favorable effect on adverse event have not been studied well. In this study, we aimed to investigate the effect of EPA and statins focusing on plaque formation in a rabbit plaque model. Methods: Rabbit carotid artery was injured with a balloon followed by feeding with a normal diet for 4 $\mathrm{w}$ and then with a high-cholesterol diet for $4 \mathrm{w}$. The rabbits were either received pitavastatin alone, EPA alone, combination (pitavastatin+EPA) or vehicle (control) for $4 \mathrm{w}$ with a high-cholesterol diet. The injured carotid arteries were harvested and analysed morphometrically and immunohistochemically. Results: In control group, smooth muscle cells were located on the luminal side of the plaque and lipidrich macrophages accumulated in the center of the plaque expressing Matrix Metalloproteinase-9 (MMP-9), Tissue Factor (TF), and NF-kB, those are characteristic of unstable plaques. In contrast, EPA reduced the expression of TF and NF-kB and increased the expression of PPAR- $\alpha$ in the plaque. Pitavastatin also reduced the expression of MMP-9, TF, and NF-kB, but not PPAR- $\alpha$ in the plaque. Combination of pitavastatin and EPA significantly reduced the expression of MMP-9, TF, and NF-kB increasing the expression of PPAR- $\alpha$ resulting in the inhibition of intimal hyperplasia and plaque formation in carotid arteries.

Conclusions: Pitavastatin and EPA synergistically played a role for inhibitory effect on plaque formation decreasing the expression of inflammation-related molecules and increasing the expression of PPAR- $\alpha$ in injured artery.
\end{abstract}

Keywords: Eicosapentaenoic acid, Statin, Plaque, Vascular smooth muscle cells.

Accepted on May 28, 2018

\section{Introduction}

Dyerberg et al. reported that many Inuit is low death rate by myocardial infarction for they consume food which fish oil including omega-3 Polyunsaturated Fatty Acid (PUFA) [1]. After then, 30-year follow-up of the Chicago Western Electric Study reported that it studies indicate secondary prevention effect to reduced risk of total mortality when patient of myocardial infarction many take fish or PUFA. Platelet Aggregation Inhibitor (PAI) is making form PUFA in Eicosapentaenoic Acid (EPA) or Docosahexaenoic Acid (DHA). We use the PAI as highly purified ethyl esterification drug. EPA has effect such as reduce of neutral fat, antiarrhythmic, anti-thrombotic, anti-inflammatory, vasodilation and anti-platelet [2]. HMG-CoA reductase inhibitor (statin) has effect to reduce cholesterol biosynthesis and serum LDL cholesterol in blood, it proved that statin is useful drug as firstly and secondary prevention to coronary heart disease by results of many clinical trials [3-5]. Recently, it attracted a lot of attention that statin has pleiotropic effects except for cholesterol reduce activity. Concretely, statin show improve of intravascular the function, reduction of macrophage activity, anti-inflammatory and anti-oxidant. Those activities seem to explain excellent achievement on clinical trial [6,7]. It reported that HMG-CoA reductase inhibitor and fish oil/PUFA of combination treatment is useful [8,9]. In 2007, the Japan Eicosapentaenoic Acid Lipid Intervention Study (JELIS) reported random and open-label endpoint blind clinical trial by EPA treatment for long period.

The JELIS study says significant reduction in coronary event, it means that the combination treatment has primary and 
secondly prevention effect [10]. Accordingly JELIS' report, EPA has different mechanism from statin single treatment to stabilization activity of plaque. The result suggests that it has strongly stabilization activity of plaque by synergism effect with combination treatment. However, the molecular mechanism has still unclear. We established model for plaque transfer stable to unstable state by balloon injury and high cholesterol food to rabbit. The purpose of this study is to elucidate plaque stabilization activity and the molecular mechanism of EPA by model rabbit with statin.

\section{Materials and Methods}

\section{Animal experimental protocols}

Institutional Animal Care and Use Committee in Osaka Medical College approved all the following research protocols, including the surgical procedures and animal care. Twentyeight male Japanese white rabbits ( 2.5 to $3.0 \mathrm{~kg}$ body weight) were obtained from Kitayama Labes, Nagoya, Japan. Animals were housed at room temperature with a $12 \mathrm{~h}$ light-dark cycle and had access to water throughout the experimental period.

Experimental atheromatous plaque was introduced by combination of balloon injury and cholesterol diet as previously reported [11]. Briefly, the rabbit's right carotid artery was injured by a balloon catheter under anesthetization with pentobarbital. A $2.75 \mathrm{~mm}$ PTCA balloon catheter $(4 \mathrm{~F}$ PCI catheter; Terumo, Tokyo) was introduced into the right external carotid artery and passed three times through the main carotid artery with the balloon distended sufficiently with air to generate slight resistance and the inflation pressures (6 atm) kept constant.

The first $4 \mathrm{w}$ of a normal standard diet and next $8 \mathrm{w}$ of a $0.5 \%$ high cholesterol diet (Oriental Yeast Co., Osaka, Japan) were fed. The rabbits were divided into the following 4 groups; 1) vehicle of $1 \%$ carboxymethyl-cellulose $(1 \mathrm{ml} / \mathrm{kg} / \mathrm{d}, \mathrm{n}=7)$ was administered orally via a gastric tube during the last 4 w. 2) pitavastatin was administered $(0.1 \mathrm{mg} / \mathrm{kg} / \mathrm{d}, \mathrm{n}=7)$ in $1 \%$ carboxymethyl-cellulose orally during the last 4 weeks. 3) Eicosapentanoic Acid (EPA) was administered $(600 \mathrm{mg} / \mathrm{kg} / \mathrm{d}$, $\mathrm{n}=7)$ in $1 \%$ carboxymethyl-cellulose $(1 \mathrm{ml} / \mathrm{kg} / \mathrm{d})$ orally during the last 4 w. 4) Both pitavastatin $(0.1 \mathrm{mg} / \mathrm{kg} / \mathrm{d})$ and EPA $(600$ $\mathrm{mg} / \mathrm{kg} / \mathrm{d}$ ) were administered orally during the last $4 \mathrm{w}$. Blood samples were collected thereafter, and total plasma cholesterol and triglycerides were measured enzymatically (SRL, Inc., Tokyo, Japan). At the end of the protocol, the rabbits were euthanized by an intravenous injection of an overdose of sodium pentobarbital. The carotid arteries were perfusion-fixed with $4 \%$ paraformaldehyde, sectioned at $5 \mathrm{~mm}$, fixed again with either $4 \%$ paraformaldehyde and embedded in paraffin. For immunohistochemical analysis with antibodies for NF-kB and PPAR- $\alpha$, carotid arteries were harvested on day 7 after the treatments following the same protocol as described above.

\section{Primary antibodies}

The primary antibodies comprised mouse monoclonal antibodies against rabbit macrophages (200:1, RAM11; Dako); $\alpha$-Smooth Muscle (SM) actin (300:1, f1A4; Sigma-Aldrich); Matrix Metalloproteinase (MMP)-9 (1:100, 56-2A4; Daiichi Fine Chemicals, Toyama, Japan); Tissue Factor (TF) (1:100, American Diagnostica, Inc.); Nuclear Factor-kappa B (NF-kB) (1:400, CST Japan); and peroxisome proliferator-activated receptor alpha (PPAR- $\alpha)$ (1:200, Merk Millipore).

\section{Histological examination}

Paraffin-embedded tissues were sectioned at $3 \mu \mathrm{m}$ and visualized by hematoxylin/eosin staining. Some sections were analysed immunohistochemically using a labeled streptavidinbiotin system. Antigen retrieval by heating in Target Retrieval Solution (code No. S3307, Dako Cytomation, Glostrup, Denmark) was added to the procedure to visualized TF.

Two experts (T.N. and K.A.) blinded to the experimental regimes performed the histopathological analyses. Planimetry was performed with digitized images at $40 \mathrm{X}$ magnification (Micro Computer Imaging Device; Imaging Research Co., Canada). Absolute histomorphometric measurements included the luminal area, the area bounded by the internal elastic lamina, and the area bounded by the external elastic lamina (overall vessel size). Macrophage and vascular SMC contents in the intimal area were calculated as ratios (\%) of the RAM11positive area/intimal area and of the $\alpha-\mathrm{SM}$ actin-positive area/ intimal area using NIH ImageJ software, respectively. MMP-9, TF, and PPAR- $\alpha$ expressions were calculated in immunohistochemical staining as ratios (\%) of MMP-9, TF, and PPAR- $\alpha$ positive area/intimal area using a NIH ImageJ software, respectively. NF-kB positivity was calculated as percent of total nuclei in the intima.

\section{Serum lipid levels}

Blood was collected from the femoral artery under general anesthesia when the rabbits were injured and from the ear artery when they were sacrificed. The serum total cholesterol and triglyceride levels were measured with a Hitachi 7180 auto-analyzer using reagents (Wako Pure Chemical Industries, Osaka, Japan).

\section{Statistical analysis}

All values are expressed as the means \pm s.e.m. Statistical analyses were performed using the PrismTM software program (GraphPad Software, CA, USA). Non-parametric unpaired ttests (Mann-Whitney U-test) were used for comparisons between two groups, and repeated-measure One-way ANOVA with Tukey post hoc tests were used for comparing multiple groups. $\mathrm{P}$ values less than 0.05 were considered statistically significant. Relationships of interest were determined using Pearson's correlation analysis. A value of $p<0.05$ was considered to indicate statistical significance. 


\section{Results}

\section{Serum lipid levels}

All animals survived through the study. Table 1 shows serum lipid profiles in each rabbit received with vehicle, pitavastatin, EPA, and combination of pitavastatin and EPA. After $8 \mathrm{w}$ on the $0.5 \%$ high-cholesterol diet, serum total cholesterol increased, whereas serum triglycerides remained unchanged in vehicle administered group. No significant change of serum total cholesterol levels in pitavastatin, EPA, and combination of pitavastatin and EPA group compared with that in vehicle group, suggesting that both pitavatsatin and EPA did not affect cholesterol metabolism. Similarly, triglyceride was not significantly changed by administration of pitavastatin, EPA, or combination of pitavastatin and EPA (Table 1).

Table 1. Lipid profiles in each group.

\begin{tabular}{llll}
\hline & $\begin{array}{l}\text { Total cholesterol } \\
(\mathbf{m g} / \mathrm{dL})\end{array}$ & $\begin{array}{l}(\mathbf{m M}) \\
\end{array}$ & $\begin{array}{l}\text { Trygryceride } \\
(\mathbf{m g} / \mathbf{d L})\end{array}$ \\
\hline Veshicle $(\mathrm{n}=7)$ & $28.7 \pm 2.64(1112 \pm 102)$ & $0.36 \pm 0.18(32 \pm 16)$ \\
\hline Pitavastatin $(n=7)$ & $31.9 \pm 0.91(1236 \pm 135)$ & $0.32 \pm 0.16(28 \pm 14)$ \\
\hline EPA $(n=7)$ & $27.6 \pm 4.55(1068 \pm 176)$ & $0.24 \pm 0.11(21 \pm 10)$ \\
\hline Combination $(n=7)$ & $31.7 \pm 1.79(1225 \pm 69)$ & $0.92 \pm 0.51(82 \pm 45)$ \\
\hline
\end{tabular}

\section{Inhibitory effect of pitavastatin and EPA on neointima formation in injured artery with high- cholesterol diet}

After balloon injury followed by $4 \mathrm{w}$ on the normal diet, rabbits were fed with high-cholesterol diet for $8 \mathrm{w}$ including for the last 4 weeks with each treatment. The evident neointima formations were observed in vehicle group. Although pitavastatin or EPA alone reduced the neointima formation, the extent was not significant statistically. In contrast, combination treatment of pitavastatin and EPA significantly reduced the neointima formation in injuries carotid arteries with highcholesterol diet (Figure 1).
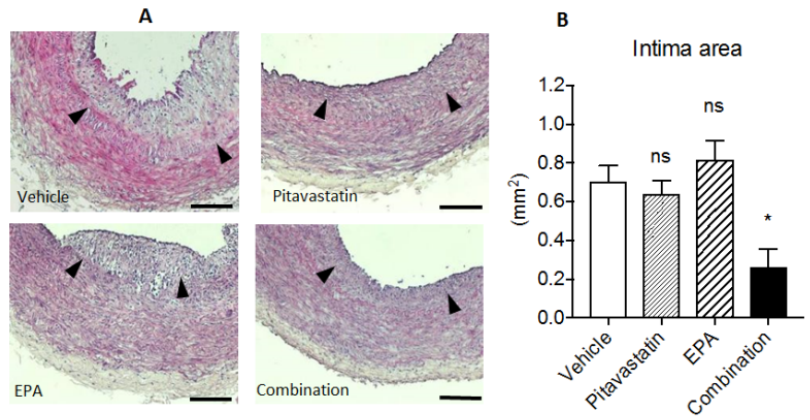

Figure 1. Inhibitory effect of pitavastatin and EPA on neointima formation.

Immunohistochemical analyses with antibodies for Vascular Smooth Muscle Cell (VSMC) and macrophage exhibited the macrophage-rich intima, specifically, in core site with thin
VSMC layer (fibrous cap) in the luminal side of intima. Pitavastatin alone also formed VSMC-rich neointima with few macrophage infiltrations, and EPA treatment alone formed VSMC-rich neointima with remarkable macrophage infiltration. The combination treatment of pitavastatin and EPA significantly reduced neointima formation with less VMSCs and few macrophage infiltration (Figure 2).

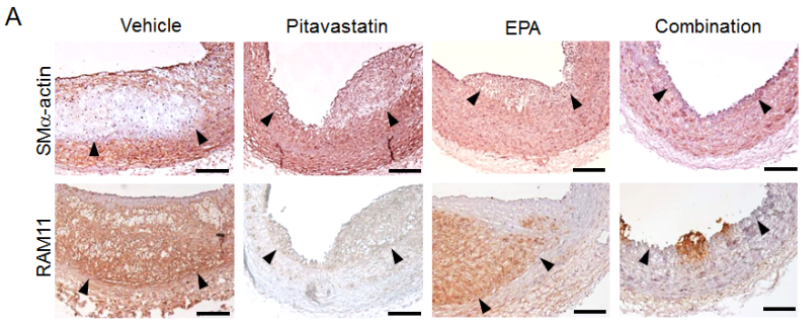

B

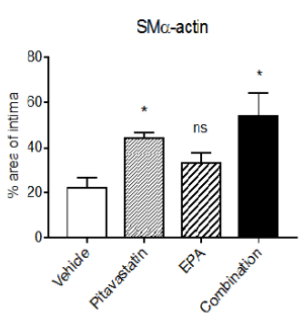

C

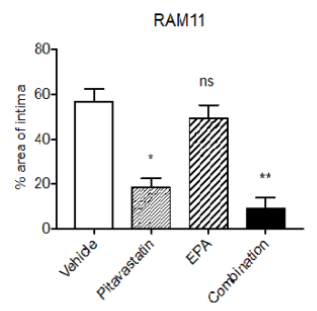

Figure 2. Immunohistochemical analyses with antibodies for vascular smooth muscle cell.
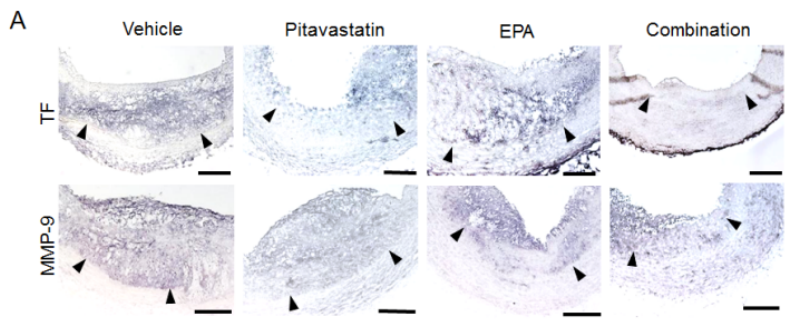

B

C
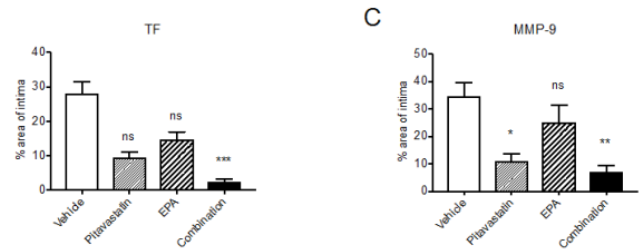

Figure 3. Regulation of plaque formation-related molecule expressions in MMP-9 and TF.

\section{Regulation of plaque formation-related molecule expressions in injured carotid arteries with high- cholesterol diet}

The formed plaque/neointima highly expressed MMP-9, TF, and NF-kB, but not PPAR $\alpha$ in vehicle group (Figures 3 and 4). Pitavastatin significantly reduced the expressions of TF and MMP-9 and weakly reduced that of NF-kB, however, PPAR- $\alpha$ 
was not up-regulated by pitavastatin alone. EPA significantly reduced the expressions of TF and NF-kB, but not MMP-9 and significantly up-regulated PPAR- $\alpha$ expression. The combination of pitavastatin and EPA treatment remarkably reduced the plaque/neointima formation down-regulating TF, MMP-9, and NF-kB expressions and up-regulating PPAR- $\alpha$ expression. When the expression of PPAR $\alpha$ and that of NF-kB were analysed statistically, there was an inverse correlation between the positivity of PPAR $\alpha$ and that of NF-kB in the plaque/neointima (Figure 5). In order to understand the histopathological characteristics easily in each experimental group, the content of VdSMCs/macrophages and the extent of TF, MMP9, NF-kB, and PPAR- $\alpha$ expressions were roughly indicated in Table 2 and the exact values for quantification of these parameters/elements in the formed plaque/neointima are also shown in Table 3.

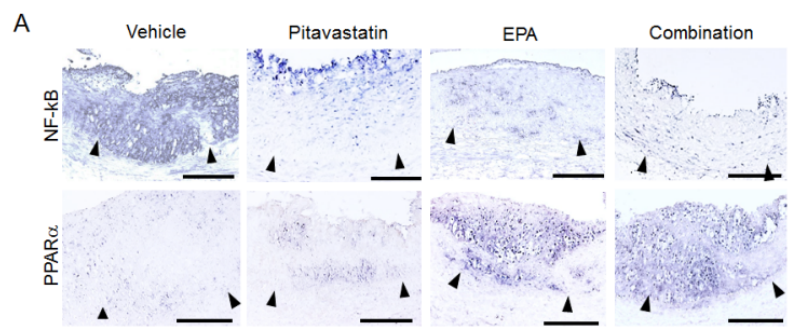

B

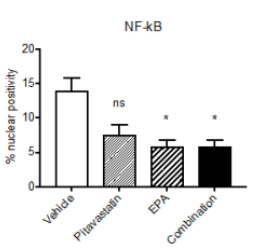

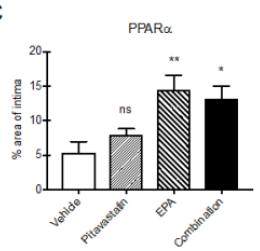

Figure 4. Regulation of plaque formation-related molecule expressions in $N F-k B$ and PPAR- $\alpha$.

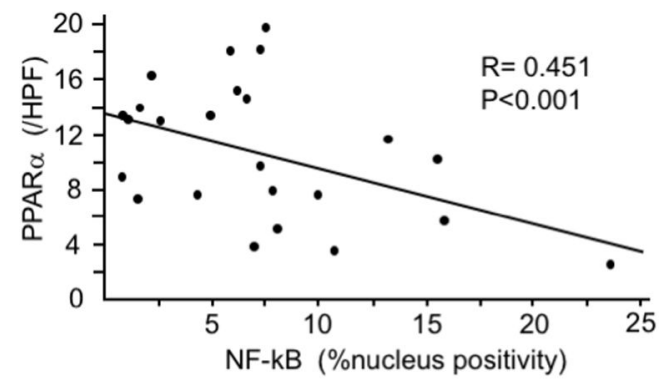

Figure 5. Correlation between the positivity of PPAR- $\alpha$ and that of $N F-k B$ in the plaque/neointima.

These findings suggest that pitavastatin potently stabilized plaque reducing macrophage accumulation. On the other hand, EPA tends to stabilize plaque up-regulating PPAR- $\alpha$. In addition, the combination of pitavastatin and EPA exhibited little plaque reducing neointima formation compared with the other groups.

\section{Discussion}

In the present study, we examined the effect of EPA, pitavastatin, and the combination of EPA and pitavastatin on atherosclerotic plaque/neointima formation histologically using a rabbit carotid balloon injury model with high-cholesterol diet, which we recently developed for investigating atherosclerotic lesion (plaque) [11].

One of the major reasons of Acute Coronary Syndrome (ACS) is known to be rupture of unstable plaque in atherosclerotic lesion. The unstable plaque has thin fibrous cap which is compose of extracellular matrix such as VSMCs and collagen, and the lipid core is under the fibrous cap with many macrophages and lipid. The lipid core is also known to express protease of MMPs and TF secreted from activated foamed VSMCs [12]. The rabbit carotid balloon injury model with high-cholesterol diet we used in this study exhibited similar pathological characteristics to human unstable plaque i.e. thin VSMC layer expressing MMP-9 and TF on luminal side (vehicle treatment group).

HMG-CoA reductase inhibitors (statins) not only have serum cholesterol lowering effect but also exhibit pleiotropic effect, for instance, inhibitory effect of VSMC proliferation, regulation of MMP-1,2,3,9 and Tissue Factor (TF) gene expression [13-15].

Table 2. Summary of results from each model.

\begin{tabular}{lllll}
\hline & Vehicle & Pitavastatin & EPA & Combination \\
\hline SMC & $+/-$ & ++ & + & $+/-$ \\
\hline Macrophage & ++++ & $+/-$ & ++ & + \\
\hline TF & +++ & + & + & $+/-$ \\
\hline MMP-9 & ++++ & + & ++ & + \\
\hline NF-kB & ++++ & + & + & + \\
\hline PPAR- $\alpha$ & $+/-$ & + & ++ & +++
\end{tabular}

SMC: Smooth Muscle Cell; TF: Tissue Factor; MMP-9: Matrix Metalloprotease 9; PPAR- $\alpha$ : Peroxisome Proliferator-activated Receptor- $\alpha$; NF-kB: Nuclear Factor-kappa B.

Indeed, we confirmed that pitavastatin decreased intimal area, perhaps, inhibiting VSMC proliferation and might also modulating phenotypic change from synthetic/dedifferentiation type to contractile/differentiation type. Pitavastatin potently inhibited macrophage infiltration into lipid core resulting in stable plaque formation in our data. Suzuki et al. reported similar result, but it was attributed to lipid-lowering. Since pitavastatin does not affect to serum lipid (total cholesterol, TG) levels when it is administered to animals at low concentration of $0.1 \mathrm{mg} / \mathrm{kg} / \mathrm{d}$ for short period (4 w), [16], we followed the experimental condition in our study. 
Table 3. Morphometrical and quantitative immunohistochemical analysis of plaques.

\begin{tabular}{|c|c|c|c|c|c|c|c|}
\hline Group & Intima & SMC & Macrophage & TF & MMP-9 & PPAR $\alpha$ & NF-kB \\
\hline Vehicle $(n=7)$ & $0.71 \pm 0.081$ & $22 \pm 3.9$ & $57 \pm 5.1$ & $28 \pm 3.5$ & $34 \pm 5.0$ & $5.3 \pm 1.7$ & $6.2 \pm 2.8$ \\
\hline Pitavastatin $(n=7)$ & $0.64 \pm 0.065$ & $45 \pm 2.1^{\star *}$ & $19 \pm 3.4^{\star \star \star}$ & $9.6 \pm 1.4^{\star *}$ & $11 \pm 2.7^{\star \star}$ & $7.9 \pm 0.89$ & $5.0 \pm 2.1$ \\
\hline $\operatorname{EPA}(n=7)$ & $0.82 \pm 0.097$ & $33 \pm 4.4$ & $50 \pm 5.2$ & $15 \pm 2.3^{*}$ & $25 \pm 6.4$ & $15 \pm 2.1^{* *}$ & $2.3 \pm 0.95^{\star \star}$ \\
\hline Combination $(n=7)$ & $0.30 \pm 0.072^{*} \dagger$ & $55 \pm 9.4^{\star *}$ & $9.6 \pm 4.4^{* * *}$ & $2.3 \pm 0.91^{* * *}+\dagger$ & $7.0 \pm 2.6^{* *}$ & $13 \pm 1.8^{* *}$ & $2.73 \pm 0.82^{* * *}$ \\
\hline
\end{tabular}

${ }^{*} \mathrm{p}<0.05$ vs. Vehicle, ${ }^{* *} \mathrm{p}<0.01$ vs. Vehicle, ${ }^{* * *} \mathrm{p}<0.001$ vs. Vehicle ${ }^{\dagger} \mathrm{P}=0.005$ vs. Pitavastatin, ${ }^{\dagger} \mathrm{P}=0.003$ vs. Pitavastatin, MMP: Matrix Metalloproteinase; PPAR: Peroxisome Proliferator-activated Receptor; NF-kappa B: Nuclear Factor kappa B; Intima: $\left(\mathrm{mm}^{2}\right)$, SMC, Macrophage, TF, and MMP9: \% of area in intima, NF-kB: \% of nucleus positivity, PPARa: /HPF

Like statins, EPA has also been reported to have a pleiotropic effect such as favorable effect of anti-arrhythmia, antithrombosis, anti-inflammation, vasodilation, and plaque stabilization including serum lipid level-lowering activity, specifically, for triglyceride. Regarding the effect of antiatherosclerosis, a previous report demonstrated that EPA reduced the number of macrophage and made stable fibrous cap with high density of collagen in unstable plaque [17]. Our results also support the findings in the previous report [17] by showing the reduction of macrophage infiltration along with reduced NF-kB expression and increased PPAR- $\alpha$ expression with thick fibrous cap composed of VSMCs in plaque. PPAR- $\alpha$ is known to deeply relate to pathology of hyperlipidemia, diabetes, and obesity. PPAR- $\alpha$ is observed on tissue having high ability of fatty acid catabolism such as liver, kidney, skeletal muscle and brown cell. EPA and fibrates are known as exogenous ligands. The both ligands enhance PPAR- $\alpha$ activity and then promote fatty acid oxidation on liver leading to efficient lipolysis [18]. Apart from the effect of PPAR- $\alpha$ on lipid metabolism, PPAR- $\alpha$ has a direct inhibitory effect on VSMC proliferation by inducing p16INK4a [19] and can induce cell cycle arrest inhibiting Transforming Growth Factor-beta1 (TGF- $\beta 1$ ) induction in SMCs [20]. These evidences might relate to a certain mechanistic insight of our findings.

$\mathrm{NF}-\mathrm{kB}$ is one of the important transcription factors which relate to the regulation of inflammatory cytokine production. An inflammation-related product of Reactive Oxygen Species (ROS) can activate NF-kB followed by gene up-regulation of inflammatory cytokine/chemokine [14]. PPAR- $\alpha$ is reported to be inhibit NF-kB activity interacting with p65 of NF-kB [21]. Sata et al. also reported that EPA treatment suppressed phosphorylation of NF-kB via a PPAR- $\alpha$ activation pathway [22]. Indeed, we found significantly increased expression of PPAR- $\alpha$ and decreased expression of NF-kB in EPA treatment group exhibiting an inverse co-relationship between the expressions of PPAR- $\alpha$ and NF-kB. Although it is known that pitavastatin treatment increase PPAR- $\alpha$ activity and decrease NF-kB activity, our results showed that the effect of pitavasatin treatment alone was not sufficient in terms of antiatherosclerosis.

In conclusion, considering the above findings and interpretation, the combination of pitavastatin and EPA treatment is thought to compensate their weak effectiveness on plaque/neointima formation each other. Namely, pitavastatin which has potent anti-inflammatory effect and mild VSMC inhibitory effect on plaque formation and EPA which has mild anti-inflammatory effect and potent suppressive effect on VSMC activation synergistically exhibited a favorable effect on atherosclerosis. As suggested in clinical study JELIS (Japan EPA Lipid Intervention Study), EPA might have an original pharmacological action on coronary artery event. Nevertheless, our results also suggest that PPAR- $\alpha$ activation in arterial (medial) VSMCs could be one of the major molecular mechanisms for anti-atherosclerotic effect of EPA in combination with statin therapy for cardiovascular diseases.

\section{Acknowledgement}

We thank Mrs. Eiko Kohbayashi for her technical assistance of tissue sectioning and immunohistochemistry. We appreciate Prof. Nobukazu Ishizaka and Prof. Toshiaki Hanafusa for their kind advice.

\section{References}

1. Bang HO, Dyerberg J, Sinclair HM. The composition of the Eskimo food in north western Greenland. Am J Clin Nutr 1980; 33: 2657-2661.

2. Kris-Etherton PM, Harris WS, Appel LJ. Fish consumption, fish oil, omega-3 fatty acids, and cardiovascular disease. Circulation 2002; 106: 2747-2757.

3. Scandinavian Simvastatin Survival Study Group. Randomised trial of cholesterol lowering in 4444 patients with coronary heart disease: the Scandinavian Simvastatin Survival Study (4S). Lancet 1994; 344: 1383-1389.

4. Shepherd J, Cobbe SM, Ford I, Isles CG, Lorimer AR. Prevention of coronary heart disease with pravastatin in men with hypercholesterolemia. N Engl J Med 1995; 333: 1301-1307.

5. Downs JR, Clearfield M, Weis S. Primary prevention of acute coronary events with lovastatin in men and women with average cholesterol levels: results of AFCAPS/ TexCAPS. Air Force/Texas Coronary Atherosclerosis Prevention Study. JAMA 1998; 279: 1615-1622.

6. Bonetti PO, Lerman LO, Napoli C. Statin effects beyond lipid lowering-are they clinically relevant? Eur Heart J 2003; 24: 225-248. 
7. Laufs U, Liao JK. Isoprenoid metabolism and the pleiotropic effects of statins. Curr Atheroscler Rep 2003; 5: 372-378.

8. Meyer BJ, Hammervold T, Rustan AC. Dose-dependent effects of docosahexaenoic acid supplementation on blood lipids in statin-treated hyperlipidaemic subjects. Lipids 2007; 42: 109-115.

9. Barter P, Ginsberg HN. Effectiveness of combined statin plus omega-3 fatty acid therapy for mixed dyslipidemia. Am J Cardiol 2008; 102: 1040-1045.

10. Yokoyama M, Origasa H, Matsuzaki M, Matsuzawa Y, Saito Y. Effects of eicosapentaenoic acid on major coronary events in hypercholesterolaemic patients (JELIS): a randomised open-label, blinded endpoint analysis. Lancet 2007; 369: 1090-1098.

11. Okabe T, Hoshiga M, Negoro N, Nakakoji T, Arishiro K. Rabbit plaque models closely resembling lesions in human coronary artery disease. Int J Cardiol 2011; 147: 271-277.

12. Falk E, Shah PK, Fuster V. Coronary plaque disruption. Circulation 1995; 92: 657-671.

13. Fukumoto Y, Libby P, Rabkin E. Statins alter smooth muscle cell accumulation and collagen content in established atheroma of watanabe heritable hyperlipidemic rabbits. Circulation 2001; 103: 993-999.

14. Luan Z, Chase AJ, Newby AC. Statins inhibit secretion of metalloproteinases-1, $-2,-3$, and -9 from vascular smooth muscle cells and macrophages. Arterioscler Thromb Vasc Biol 2003; 23: 769-775.

15. Mitani H, Egashira K, Kimura M. HMG-CoA reductase inhibitor, fluvastatin, has cholesterol-lowering independent direct effects on atherosclerotic vessels in high cholesterol diet-fed rabbits. Pharmacol Res 2003; 48: 417-427.

16. Kitahara M, Kanaki T, Toyoda K, Miyakoshi C, Tanaka S. NK-104, a newly developed HMG-CoA reductase inhibitor, suppresses neointimal thickening by inhibiting smooth muscle cell growth and fibronectin production in balloon-injured rabbit carotid artery. JPN J Pharmacol 1998; 77: 117-128.
17. Suzuki H, Kobayashi H, Sato F, Yonemitsu Y, Nakashima Y. Plaque-stabilizing effect of pitavastatin in Watanabe heritable hyperlipidemic (WHHL) rabbits. J Atheroscler Thromb 2003; 10: 109-116.

18. Tsuboyama-Kasaoka N, Takahashi M, Kim H, Ezaki O. Up-regulation of liver uncoupling protein-2 mRNA by either fish oil feeding or fibrate administration in mice. Biochem Biophys Res Commun 1999; 257: 879-885.

19. Gizard F, Amant C, Barbier O, Bellosta S, Robillard R. PPAR alpha inhibits vascular smooth muscle cell proliferation underlying intimal hyperplasia by inducing the tumor suppressor p16INK4a. J Clin Invest 2005; 115: 3228-3238.

20. Lien SC, Wei SY, Chang SF, Chang MD, Chang JY. Activation of PPAR-alpha induces cell cycle arrest and inhibits transforming growth factor-beta1 induction of smooth muscle cell phenotype in 10T1/2 mesenchymal cells. Cell Signal 2013; 25: 1252-1263.

21. Delerive P, De Bosscher K, Besnard S, Vanden Berghe W, Peters JM. Peroxisome proliferator-activated receptor alpha negatively regulates the vascular inflammatory gene response by negative cross-talk with transcription factors NF-kappaB and AP-1. J Biol Chem 1999; 274: 32048-32054.

22. Matsumoto M, Sata M, Fukuda D, Tanaka K, Soma M. Orally administered eicosapentaenoic acid reduces and stabilizes atherosclerotic lesions in ApoE-deficient mice. Atherosclerosis 2008; 197: 524-533.

\section{*Correspondence to}

Masaaki Ii

Division of Research Animal Laboratory and Translational Medicine

Research and Development Center

Osaka Medical College

Japan 